of $\$ 13,901$ per SSI compared with patients who had MSSA SSIs. The authors concluded that methicillin resistance is independently associated with increased mortality and hospital charges among patients with $S$. aureus SSI.

FROM: Engemann JJ, Carmeli Y, Cosgrove SE, et al. Adverse clinical and economic outcomes attributable to methicillin resistance among patients with Staphylococcus aureus surgical site infection. Clin Infect Dis 2003;36:592598.

\section{Laparoscopic Cholecystectomy and the Risk of Surgical-Site Infection}

Richards and colleagues from the National Nosocomial Infections Surveillance (NNIS) System of the Centers for Disease Control and Prevention conducted a study to assess the impact of laparoscopy on surgical-site infections (SSIs) following cholecystectomy in a large population of patients. Previous investigations have demonstrated that laparoscopic cholecystectomy is associated with a shorter postoperative stay and fewer overall complications. Less is known about the impact of laparoscopy on the risk for SSIs.

An epidemiologic analysis was performed on data collected during a 7-year period (1992 to 1999) by hospitals participating in the NNIS System. For 54,504 inpatient cholecystectomy procedures reported, use of the laparoscopic technique increased from 59\% in 1992 to $79 \%$ in 1999. The overall rate of SSI was significantly lower for laparoscopic cholecystectomy than for open cholecystectomy. Overall, infecting organisms were similar for both approaches. Even after other significant factors were controlled, the risk for SSI was lower in patients undergoing the laparoscopic technique than in those undergoing the open technique.

The authors concluded that laparoscopic cholecystectomy is associated with a lower risk for SSI than is open cholecystectomy, even after adjusting for other risk factors. For interhospital comparisons, SSI rates following cholecystectomy should be stratified by the type of technique.

FROM: Richards C, Edwards J, Culver D, Emori TG, Tolson J, Gaynes R. Does using a laparoscopic approach to cholecystectomy decrease the risk of surgical site infection? Ann Surg 2003;237:358-362.

\section{Blood Culture Contamination: Dedicated Phlebotomy Versus Intravenous Catheter}

Blood culture is the criterion standard for identifying children with bacteremia. However, elevated false-positive rates are common and are associated with substantial healthcare costs.

Norberg and colleagues recently compared contamination rates in blood culture specimens obtained from separate sites versus through newly inserted intravenous catheters in an observational study conducted from January 1998 through December 1999. Patients included those 18 years or younger who were seen in a U.S. children's hospital emergency department and had a blood culture performed as part of their care. Medical records were reviewed for all cases with a positive blood culture. Patients with indwelling vascular catheters were excluded.

Phlebotomy was performed by registered nurses of the emergency department. During the baseline phase, blood specimens for culture were obtained simultaneously with intravenous catheter insertion. During the postintervention phase, specimens were obtained by a separate, dedicated procedure. The contamination rate in the postintervention period was compared with that in the baseline period.

A total of 4,108 blood cultures were evaluated, including 2,108 during the baseline phase and 2,000 in the postintervention phase. The false-positive blood culture rate decreased from $9.1 \%$ to $2.8 \%(P<.001)$. A statistical process control chart demonstrated a steady-state process in the baseline phase and the establishment of a significantly improved steady state in the postintervention phase. Young age was associated with an increased contamination rate in both the baseline and the postintervention periods.

The authors concluded that blood culture contamination rates were lower when specimens were drawn from a separate site compared with when they were drawn through a newly inserted intravenous catheter.

FROM: Norberg A, Christopher NC, Ramundo ML, Bower JR, Berman SA. Contamination rates of blood cultures obtained by dedicated phlebotomy vs intravenous catheter. JAMA 2003;289:726-729. 